\title{
Efeito da poda radicular e do hidrogel no crescimento de mudas de jatobá
}

\author{
Bruno Vilela FALLER ${ }^{1}$, Rhayssa dos Santos AMORIN ${ }^{1}$, Israel Alves de OLIVEIRA², \\ Iselino Nogueira JARDIM ${ }^{1 *}$
}

\author{
${ }^{1}$ Faculdade de Engenharia Florestal, Universidade Federal do Pará, Altamira, PA, Brasil. \\ (Orcid: 0000-0001-5543-9588; 0000-0001-8780-3049) \\ ${ }^{2}$ Instituto de Desenvolvimento Florestal de Biodiversidade do Estado do Pará, Belém, PA, Brasil. \\ (Orcid: 0000-0003-3690-4987) \\ *E-mail: iselinojardim@gmail.com (Orcid: 0000-0001-5159-1654)
}

\begin{abstract}
Recebido em 14/05/2020; Aceito em 02/06/2020; Publicado em 31/07/2020.
RESUMO: A produção de mudas de jatobá (Hymenaea courbaril L.) é essencial para a conservação e restituição ao seu habitat natural. Entretanto a produção de mudas de espécie nativa ainda é pouco estudada. Portanto, o objetivo deste estudo foi avaliar o efeito de diferentes níveis de poda e doses de hidrogel no crescimento inicial de jatobá sob sombreamento. Mudas de jatobá com 90 dias de idade foram usadas no experimento. O delineamento experimental foi o esquema fatorial composto de três níveis de poda radicial $(0,0 ; 25,0$ e 50,0\%) e cinco doses de hidrogel $(0,0 ; 2,0 ; 4,0 ; 6,0$ e 8,0 g L-1 de substrato) totalizando 15 tratamentos. Aos 105 dias após o transplantio em casa de vegetação, foram avaliados os parâmetros morfométricos e índices de crescimento. As mudas submetidas à poda radicial de 25,0\% apresentaram maior crescimento após transplantio para sacos plásticos. O hidrogel também mostrou efeito positivo nas variáveis morfométricas avaliadas. Efeitos combinados do nível de poda radicial a $25,0 \%$ e doses de hidrogel na faixa de 3,0 a 4,0 g L $\mathrm{L}^{-1}$ exerceram efeitos positivos no crescimento das variáveis morfométricas. Essas técnicas isoladas ou combinadas podem ser aplicadas por viveiristas para prolongar o tempo de manutenção de mudas de jatobá.
\end{abstract}

Palavras-chave: Polímero hidroabsorvente; déficit hídrico; sistema radicial; Hymenaea courbaril L.

\section{Effect of root pruning and hydrogel on growth of jatoba seedlings}

\begin{abstract}
The production of jatoba (Hymenaea courbaril L.) seedlings is essential for the conservation and restitution of their natural habitat. However, the production of seedlings of native species is still little studied. Therefore, the objective of this study was to evaluate the effect of different levels of pruning and hydrogel doses on the initial growth of jatoba under shading. We used jatoba seedlings with 90 days of age in a factorial scheme consisting of three levels of root pruning $(0.0,25.0$, and 50.0\%) and five hydrogel doses $(0.0,2.0,4.0$, 6.0 and $8.0 \mathrm{~g} \mathrm{~L}-1$ substrate) totalizing 15 treatments. At 105 days after transplanting in the greenhouse, morphometric parameters and growth rates were evaluated. Seedlings submitted to root pruning of $25.0 \%$ showed greater growth after transplanting to plastic bags. The hydrogel also showed a positive effect on the morphometric variables evaluated. The combined effects of $25.0 \%$ of root pruning level and doses of hydrogel in the range of 3.0 to $4.0 \mathrm{~g} \mathrm{~L}^{-1}$ exerted positive effects on the growth of morphometric variables. These isolated or combined techniques can be employed in a nursery to prolong the maintenance time of jatoba seedlings. Keywords: Hydroabsorbent polymer; water deficit; root system; Hymenaea courbaril L.
\end{abstract}

\section{INTRODUÇÃO}

Hymenaea courbaril L., da família Fabaceae, é uma espécie arbórea de ampla distribuição geográfica, ocorrendo desde o Sul do México até grande parte da América do Sul (COSTA et al., 2011). No Brasil, o jatobá pode ser encontrado do Piauí ao Norte do Paraná, podendo atingir entre 10 a $30 \mathrm{~m}$ de altura no território brasileiro (SILVA et al., 2016). O jatobá é uma espécie clímax, semidecídua, pouco exigente em fertilidade e umidade do solo em seus ambientes naturais (COSTA et al., 2011). Essa espécie destaca-se por sua importância econômica, visto que tem grande uso no setor madeireiro quanto na medicina popular. Contudo os múltiplos usos, somados à exploração inadequada de seus recursos, têm levado à redução populacional dessa espécie (GARIGLIO et al., 2010). Nesse cenário, destaca-se a importância de definir estratégias para a conservação da população de $H$. courbaril. Dentre as estratégias, aponta-se a produção de mudas que é uma das importantes etapas da silvicultura. No entanto, para o sucesso de ações de revegetação, resgate de germoplasma e estabelecimento de povoamentos, é essencial o uso de mudas de qualidade (CRUZ et al., 2016). Entretanto existe carência de estudos, para a produção de mudas de espécies nativas, a exemplo do jatobá e, portanto, um potencial objeto de pesquisa.

A produção de mudas de qualidade depende de vários fatores abióticos, sendo a água um fator de grande importância, pois está diretamente ligada ao crescimento e desenvolvimento das plantas. Todavia o uso descontrolado desse recurso natural, associado às perspectivas de escassez, têm motivado a busca por tecnologias para otimização do seu uso e de baixo custo. Dentre as tecnologias utilizadas, para o fornecimento de água às plantas, ressaltam-se os condicionadores de solo, conhecidos como polímeros hidroabsorventes ou hidrogéis agrícolas (KLEIN; KLEIN, 
2015). Os hidrogéis agrícolas são matrizes tridimensionais constituídas de polímeros hidrofílicos lineares ou ramificados, com capacidade de absorver grandes quantidades de água (GUILHERME et al., 2015). O uso desses polímeros hidroabsorventes, no entanto é pouco estudado, principalmente, para espécies nativas.

Alguns trabalhos da literatura relatam os benefícios do uso do hidrogel para as plantas. O hidrogel é adequado para a germinação e crescimento precoce de cana-de-açúcar em condições de solo arenoso de sequeiro (WATANABE et al., 2019). A aplicação de hidrogel diminuiu a frequência de irrigação de várias culturas, melhorou a capacidade de retenção de água, além de atrasar aparecimento de murcha permanente sob intensa evaporação (NEETHU et al., 2018). Navroski et al. (2014) encontraram resultados semelhantes com adição do polímero em mudas de Eucalyptus dunnit relacionado à frequência de irrigação. Azevedo et al. (2016) estudaram que o hidrogel incorporado ao substrato contribuiu para o maior crescimento e padrão de qualidade de mudas de Corymbia citriodora. Zhao et al. (2019) constataram o aumento da capacidade de armazenamento de água no solo com diminuição da evaporação da água do solo. Dranski et al. (2013) observaram o aumento na sobrevivência pós-plantio e a inexistência de efeitos negativos sobre o crescimento a posteriori das mudas de pinhão-manso.

Além da escolha de um condicionador de solo eficiente, práticas culturais podem ser feitas, para melhorar a qualidade das mudas, entre as quais se detecta a poda do sistema radicular. Todavia o uso da poda em viveiro é pouco aplicado às espécies nativas, necessitando-se de estudos para aclarar em quais espécies pode ser adotado tal procedimento (HOSSEL et al., 2014). Em geral, a poda radicular é uma técnica empregada por viveiristas e produtores de mudas para prolongar o período de permanência da muda dentro do viveiro.

Alguns autores têm demonstrado que a poda do sistema radicular pode apresentar vários benefícios para o desenvolvimento das mudas (FREITAS et al., 2009; AZEVEDO et al., 2010; CARRA et al., 2017). Por outro lado, na literatura, há poucos relatos dos efeitos negativos da poda radicular sobre a formação e desenvolvimento das mudas em nível de viveiro (CASTRO FARIA et al., 2007). Logo nota-se que a aplicação da poda radicular pode apresentar resultado contraditório, pois depende da espécie utilizada e de outros fatores ligados à produção. Portanto parece interessante observar o comportamento das mudas de jatobá quanto ao uso da poda radicular como objeto de pesquisa.

Nesse contexto, gerar informações silviculturais básicas, para o cultivo de espécies nativas, principalmente, daquelas que possuem importância econômica e ambiental, mas que estão sob risco de extinção, são bem-vindas. Assim, o estudo objetivou avaliar o efeito de diferentes níveis de poda e doses de hidrogel no crescimento inicial de jatobá sob sombreamento.

\section{MATERIAL E MÉTODOS}

O estudo foi realizado no período de junho a dezembro de 2018, em casa de vegetação do Laboratório de Plantas Medicinais (LabMed) da Universidade Federal do Pará, Campus Altamira.

Os frutos foram coletados de plantas de jatobá localizadas em área pertencente à ELETRONORTE S/A, município de Altamira-PA. O clima de Altamira é do tipo equatorial Am e Aw, da classificação de Köppen, apresentando temperaturas médias de $26{ }^{\circ} \mathrm{C}$ e precipitação mensal média de $1.700 \mathrm{~mm}$ (ALVAREZ et al., 2013). A paisagem natural apresenta predominância da Floresta Equatorial Latifoliada. No estado do Pará, 74\% dos solos são representados por Latossolos e Argissolos (SOUZA et al., 2018). Os frutos coletados foram devidamente limpos, despolpados e as sementes extraídas acondicionadas em sacos plásticos e mantidos à temperatura ambiente por 20 dias até a semeadura.

As sementes foram desinfestadas, superficialmente, pela imersão em solução de etanol a 70\% (v/v), por 30 segundos, seguida da solução de hipoclorito de sódio a $2,5 \%$ (v/v), por 60 segundos, seguida de três enxágues das sementes em água destilada esterilizada em autoclave.

Antes da semeadura direta nos recipientes, as sementes foram submetidas a tratamento pré-germinativo, lixando-as no lado oposto ao hilo. As sementes de jatobá foram semeadas, em badejas plásticas $(9,7 \times 30,0 \times 50,0 \mathrm{~cm})$ perfuradas no fundo com areia lavada, mantidas em sala climatizada $\left(24 \pm 1,0^{\circ} \mathrm{C}\right)$ e irrigadas duas vezes ao dia. As sementes germinadas foram repicadas para tubetes de polipropileno rígido com oito estrias internas com capacidade volumétrica de $280 \mathrm{~cm}^{3}(190 \mathrm{~mm}$ de altura e 52 $\mathrm{mm}$ de diâmetro interno). Os tubetes foram preenchidos com substrato fibra de coco (AMAFIBRA ${ }^{\circledR}$ ), de textura fina, indicado para uso agrícola e florestal. As mudas foram mantidas nos tubetes por 90 dias em casa de vegetação, com nível de sombreamento de 50,0\% (sombrite) e irrigadas diariamente. Após esse período, foi realizada a mensuração das variáveis de crescimento em altura da parte aérea $(\mathrm{H})$, do diâmetro do coleto (DC), do número de folhas $(\mathrm{NF})$ e da relação $\mathrm{H} / \mathrm{DC}$. Esses valores obtidos foram considerados iniciais (Xi) para as avaliações dos índices de crescimento (IC): H $(26,82 \pm 1,37), \mathrm{DC}(4,57 \pm 0,62), \mathrm{H} / \mathrm{DC}(5,98 \pm 0,90)$ e NF $(4,47 \pm 0,52)$. Logo depois, foram executados os devidos cortes do sistema radicial, sendo 0,0 (sem poda), 25,0 e $50,0 \%$ do comprimento total do sistema radicial, medidos com uma régua graduada $(\mathrm{cm})$. Após essa etapa, as mudas foram transplantadas, para sacos de polietileno de baixa densidade com furos para drenagem de dimensão $20 \times 10 \mathrm{~cm}$, que continham a mistura do hidrogel e o substrato fibra de coco seco.

O hidrogel Hydroplan-EB ${ }^{\circledR}$ utilizado consistiu em um polímero hidroabsorvente agrícola, o qual foi misturado de forma homogênea ao substrato seco. As doses foram empregadas, conforme os tratamentos planejados $(0,0 ; 2,0$; 4,0; 6,0 e 8,0 gramas de hidrogel por litro de substrato). Após esse processo, as mudas já transplantadas foram irrigadas, diariamente por aspersão, durante sete dias, em duas frações de 30 minutos (uma pela manhã e a outra à tarde). Após o período de sete dias, as mudas foram irrigadas, quinzenalmente, por aspersão, em duas frações de 30 minutos (uma pela manhã e a outra à tarde). As mudas permaneceram em casa de vegetação, durante um período de 105 dias, com nível de sombreamento de $50,0 \%$, por meio do uso de telas de polipropileno preto (sombrite).

Aos 105 dias após o transplantio e exposição das mudas aos tratamentos, foram avaliadas suas características morfométricas: H, DC e NF em seguida, particionadas em parte aérea e sistema radicular. As raízes foram lavadas em 
água corrente e colocadas dentro de sacos de papel, para secagem em estufa com circulação forçada de ar, a aproximadamente, $65^{\circ} \mathrm{C}$, até massa constante. Foram obtidas a massa seca da parte aérea (MSPA), massa seca da raiz (MSR), massa seca total (MST). Relações entre as variáveis anteriores também foram determinadas, sendo: H/DC, H/MSPA e MSPA/MSR e o Índice de Qualidade de Dickson - IQD, calculado pela Equação 1:

$$
I Q D=\frac{M S T}{\frac{H}{D C}+\frac{M S P A}{M S R}}
$$

O Delineamento experimental adotado foi em Blocos Casualizados (DBC), em esquema fatorial de $3 \times 5$, totalizando 15 tratamentos, sendo os fatores os três tipos de podas e as cinco dosagens de hidrogel com seis repetições (seis mudas por tratamento), totalizando 90 mudas.

O cálculo do índice de crescimento (IC) foi realizado em porcentagem através da Equação 2:

$$
\mathrm{IC}=\frac{\mathrm{Xf}-\mathrm{Xi}}{\mathrm{Xf}} \times 100
$$

em que: IC é o índice de crescimento em \%, o Xf e Xi são os valores médios obtidos no final e início do experimento para $\mathrm{H}, \mathrm{DC}, \mathrm{H} / \mathrm{DC}$ e NF.

Para a análise estatística, verificou-se, inicialmente, se os dados das variáveis analisadas atendiam às pressuposições básicas de normalidade através do teste de Kolmogov Smirnov e homocedasticidade com o teste de Bartlett, no programa Paleontological Statistics Software Package for Education and Data Analysis (PAST). E, sendo atendidos esses parâmetros, os resultados foram submetidos à análise de variância e Teste de Tukey, ao nível de 5\% de probabilidade e análise de regressão para as doses de hidrogel.

\section{RESULTADOS}

$\mathrm{Na}$ Tabela 1 são apresentados os valores do quadrado médio (QM) da análise de variância para as variáveis morfométricas e índices avaliados. Observou-se efeito significativo, para as fontes de variação isoladas (poda e hidrogel) nas variáveis H, DC, IQD e H/MSPA e somente o fator poda foi significativo em relação à H/DC. Houve efeito significativo da interação poda*hidrogel (P*Hid) em relação a MSPA, MSR, MST e MSPA/MSR. A variável NF não apresentou efeito significativo para nenhuma fonte de variação (Tabela 1$)$.
A Tabela 2 mostra os valores médios obtidos para as variáveis morfométricas e índices que sofreram a influência dos diferentes níveis de poda radicular. A altura foi a variável morfométrica mais afetada pela poda do sistema radicular. Mudas com podas mais leves cresceram mais que as sem poda. A poda da raiz exerceu efeito significativo na variável H. A maior média $(41,38 \mathrm{~cm})$ em $\mathrm{H}$ foi atingida com a poda de $25,0 \%$ da raiz. Enquanto a menor média $(29,0 \mathrm{~cm})$ ocorreu com a poda de $50,0 \%$ da raiz. Os índices de crescimento calculado pela Equação 2 mostra que a poda do sistema radicular de $25,0 \%$ levou a maior incremento de crescimento em $\mathrm{H}$, cerca de $35,19 \%$. Por outro lado, a poda de $50,0 \%$ da raiz levou a menor incremento de crescimento, cerca de $7,74 \%$.

Em relação ao DC, o crescimento máximo das mudas foi obtido com a poda de $25,0 \%$ da raiz, com média de $5,97 \mathrm{~cm}$, diferenciando-se estatisticamente dos demais tratamentos. Por outro lado, a menor média para o DC $(4,84 \mathrm{~cm})$ ocorreu no tratamento com a poda de 50,0\% da raiz (Tabela 2). Os índices de crescimento (Equação 2) mostra novamente que 25,0\% de poda do sistema radicular levou a maior incremento de crescimento em DC, cerca de $23,45 \%$. Por outro lado, a poda de $50,0 \%$ da raiz levou a menor incremento de crescimento, cerca de $5,58 \%$.

Tabela 2. O efeito dos diferentes níveis de poda radicular no crescimento e qualidade de mudas de Hymenaea courbaril L. aos 105 dias após o transplantio em condições de casa de vegetação, Altamira/PA.

Table 2. Effect of different root pruning levels on growth and quality of Hymenaea courbaril L. seedlings at 105 days after transplanting under greenhouse conditions, Altamira/PA.

\begin{tabular}{cccccc}
\hline Poda & H & DC & H/DC & IQD & H/MSPA \\
\hline $0,0 \%$ & $32,83 \mathrm{~b}$ & $5,39 \mathrm{~b}$ & $6,30 \mathrm{~b}$ & $0,63 \mathrm{a}$ & $10,18 \mathrm{~b}$ \\
$25,0 \%$ & $41,38 \mathrm{a}$ & $5,97 \mathrm{a}$ & $7,04 \mathrm{a}$ & $0,62 \mathrm{a}$ & $11,7 \mathrm{a}$ \\
$50,0 \%$ & $29,07 \mathrm{c}$ & $4,84 \mathrm{c}$ & $6,09 \mathrm{~b}$ & $0,51 \mathrm{~b}$ & $10,32 \mathrm{~b}$ \\
\hline DMS & 2,44 & 0,53 & 0,70 & 0,07 & 1,12 \\
\hline
\end{tabular}

Médias seguidas de mesma letra na coluna não diferem significativamente entre si, pelo teste de Tukey a 5\% de probabilidade. DMS: diferença mínima significativa.

O valor da H/DC foi mais elevado $(7,04)$ a $25,0 \%$ de poda da raiz com $15,06 \%$ de incremento no índice de crescimento, sendo bem superior aos demais tratamentos. Mudas dos tratamentos $0,0 \%$ e $25,0 \%$ de poda radicial apresentaram valores médios de IQD estatisticamente iguais entre si, porém diferentes ao tratamento a 50,0\% de poda radicial. O maior valor para $\mathrm{H} / \mathrm{MSPA}$ foi obtido para $\mathrm{O}$ tratamento $25,0 \%$ de poda da raiz, que diferenciou estatisticamente dos demais tratamentos.

Tabela 1. Análise de variância do crescimento e qualidade de mudas de Hymenaea courbaril L. aos 105 dias após o transplantio, submetidas a diferentes níveis de poda radicial e de hidrogel em condições de casa de vegetação, Altamira/PA.

Table 1. Analysis of variance of growth and quality of Hymenaea courbaril L. seedlings at 105 days after transplanting, submitted to different

\begin{tabular}{|c|c|c|c|c|c|c|c|c|c|c|c|}
\hline \multirow{2}{*}{$\begin{array}{l}\text { Fontes de } \\
\text { variação }\end{array}$} & \multirow[t]{2}{*}{ GL } & \multicolumn{10}{|c|}{ Valores do Quadrado Médio (QM) } \\
\hline & & $\mathrm{H}$ & $\mathrm{DC}$ & $\mathrm{H} / \mathrm{DC}$ & $\mathrm{NF}$ & MSPA & MSR & MST & IQD & $\mathrm{H} / \mathrm{MSPA}$ & MSPA/MSR \\
\hline Bloco & 5 & $24,07 \mathrm{~ns}$ & $0,104^{\mathrm{ns}}$ & $0,59 \mathrm{~ns}$ & $1,35^{\mathrm{ns}}$ & $0,12^{\mathrm{ns}}$ & $0,03^{\text {ns }}$ & $0,14^{\mathrm{ns}}$ & $0,00^{\mathrm{ns}}$ & $5,11^{\mathrm{ns}}$ & $0,07 \mathrm{~ns}$ \\
\hline $\mathrm{P}$ & 2 & $1194,40 *$ & $9,60 *$ & $7,57^{*}$ & $4,21^{\mathrm{ns}}$ & $3,88^{*}$ & $3,00 *$ & $13,92 *$ & $0,14^{*}$ & $21,29 *$ & $1,26 *$ \\
\hline $\mathrm{H}$ & 4 & $92,98^{*}$ & $2,10 *$ & $2,40^{\mathrm{ns}}$ & $2,63^{\mathrm{ns}}$ & $2,80 *$ & $0,98^{*}$ & $6,96^{*}$ & $0,12^{*}$ & $10,20 *$ & $0,13^{\text {ns }}$ \\
\hline $\mathrm{P} *$ Hid & 8 & $27,08 \mathrm{~ns}$ & $0,82^{\mathrm{ns}}$ & $0,37 \mathrm{~ns}$ & $0,42^{\mathrm{ns}}$ & $0,65^{*}$ & $0,18^{*}$ & $1,16^{*}$ & $0,02^{\mathrm{ns}}$ & $5,29 \mathrm{~ns}$ & $0,35^{*}$ \\
\hline Média Geral & & 34,43 & 5,40 & 6,48 & 6,31 & 3,29 & 1,63 & 4,90 & 0,59 & 10,73 & 2,06 \\
\hline $\mathrm{CV} \%$ & & 11,46 & 15,92 & 17,51 & 22,27 & 13,93 & 10,91 & 11,01 & 18,29 & 16,92 & 14,48 \\
\hline
\end{tabular}
levels of root pruning and hydrogel under greenhouse conditions, Altamira/PA. 
O crescimento em $\mathrm{H}$ apresentou resposta quadrática a doses de hidrogel, o que possibilitou estimar doses de 2,95 g $\mathrm{L}^{-1}$ para obter $\mathrm{H}$ de $36,63 \mathrm{~cm}$ (Figura $1 \mathrm{~A}$ ). Para o DC, o crescimento máximo foi estimado na dose de $4,11 \mathrm{~g} \mathrm{~L}^{-1}$ para obter a maior média de $5,75 \mathrm{~mm}$ (Figura $1 \mathrm{~B}$ ).

O IQD apresentou um ponto máximo $(0,67)$, na dose 3,89 $\mathrm{g} \mathrm{L}^{-1}$ do hidrogel, diminuindo a partir desse nível (Figura 2 A). Para a relação $\mathrm{H} / \mathrm{MSPA}$, o melhor valor estimado, na análise de regressão $(10,11)$, foi na dose de 3,95 $\mathrm{g} \mathrm{L}^{-1}$ do hidrogel (Figura 2 B).

Quando analisada a interação entre os fatores poda*hidrogel, observou-se que houve interação significativa para os parâmetros morfológicos MSPA, MSR e MST e o índice MSPA/MSR (Tabela 1). Assim, buscou-se determinar qual a combinação de poda e hidrogel que apresentasse a maior e menor média para essas variáveis.
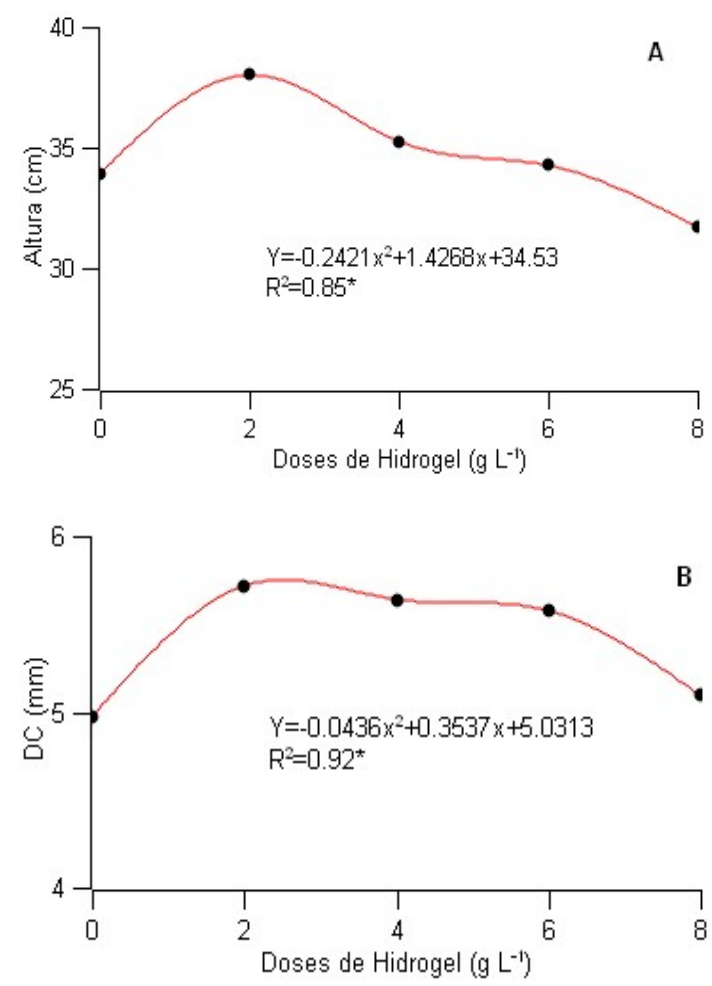

Figura 1. Altura (A) e diâmetro do coleto (B) aos 105 dias em mudas de jatobá submetidas a doses crescentes de hidrogel.

$*$ Significativo a $5 \%$ de probabilidade.

Figure 1. Height (A) and collection diameter (B) at 105 days in jatoba seedlings submitted to increasing doses of hydrogel. * Significant at $5 \%$ probability.

Para a variável MSPA, verificou-se que a melhor média (3,77 g) foi obtida na associação de $2,75 \mathrm{~g} \mathrm{~L}^{-1}$ de hidrogel + $25,0 \%$ de poda radicial. E a menor média $(3,15 \mathrm{~g})$ verificouse na combinação de $4,42 \mathrm{~g} \mathrm{~L}^{-1}$ de hidrogel $+50,0 \%$ de poda radicial (Figura $3 \mathrm{~A}$ ). Em relação à MSR, constatou-se que a maior média $(2,12 \mathrm{~g})$ ocorreu na combinação de $3,77 \mathrm{~g} \mathrm{~L}^{-1}$ de hidrogel $+25,0 \%$ de poda radicular. E a menor média $(1,33$ g) verificou-se na combinação de $2,91 \mathrm{~g} \mathrm{~L}^{-1}$ de hidrogel + $50,0 \%$ de poda radicial (Figura $3 \mathrm{~B}$ ).

No que se refere à produção de MST, foi observado que a maior média $(6,18 \mathrm{~g})$ ocorreu na combinação de $3,25 \mathrm{~g} \mathrm{~L}^{-1}$ de hidrogel $+25,0 \%$ de poda radicular. E a menor média $(4,48 \mathrm{~g})$ verificou-se na combinação de $4,38 \mathrm{~g} \mathrm{~L}^{-1}$ de hidrogel $+50,0 \%$ de poda radicial (Figura 4 A). Neste estudo, verificou-se que os valores obtidos, para MSPA/MSR das mudas de jatobá, variaram de 1,70 a 2,47 (Figura 4 B).
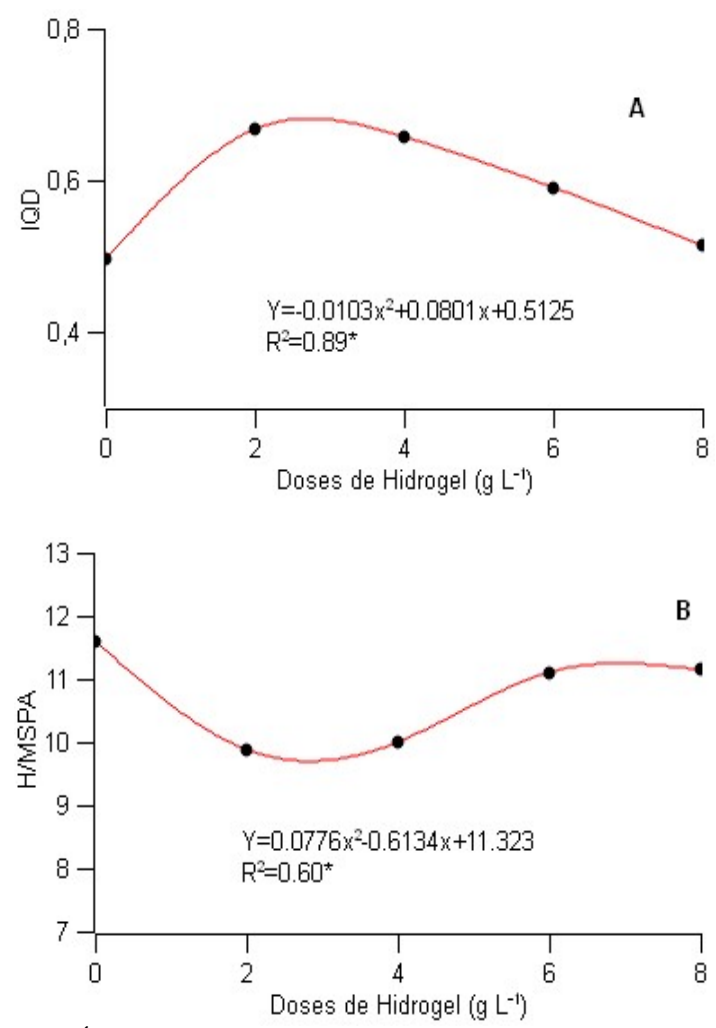

Figura 2. Índice de Dickson (A) e razão entre altura e massa seca da parte aérea (B) aos 105 dias em mudas de jatobá submetidas a doses crescentes de hidrogel. *Significativo a $5 \%$ de probabilidade.

Figure 2. Dickson's index (A) and the ratio between shoot height and dry mass (B) at 105 days in jatoba shoots submitted to increasing doses of hydrogel. *Significant at $5 \%$ probability.

\section{DISCUSSÃO}

O experimento demonstrou que os diferentes níveis de poda contribuem, porém, de forma diferente, para o crescimento das plantas de jatobá ( $p<0,05$; Tabela 2). A superioridade do tratamento de $25,0 \%$ de poda radicular aos demais tratamentos nas variáveis morfométricas avaliadas, provavelmente é devido ao maior número de pelos absorventes formados. Assim, o tratamento de 25,0\% de poda radicular é indicado para o crescimento inicial das mudas de jatobá em casa de vegetação. Pois, melhorou o desempenho das raízes e, consequentemente, o crescimento das plantas. Em contraste, o tratamento de 50,0\% de poda não é indicado com base nos decréscimos observados nas variáveis morfométricas comparadas ao controle. Isso demonstra que, provavelmente, na redução drástica das raízes, os fotoassimilados foram redirecionados para o crescimento de novas raízes (DAFLON et al., 2019). Esse fato, induz as plantas a investir parte de sua energia para essa área afetada, de modo a retornar o crescimento radicular (DAFLON et al. (2019).

O aumento na altura das mudas de jatobá mostrou a 
importância da técnica de poda das raízes. Todavia para outras espécies a técnica de poda do sistema radicular não influenciou na altura das mudas (FREITAS et al., 2009; AZEVEDO et al., 2010). Por outro lado, Alvarenga et al. (1994) observaram que as mudas de Eucaliptus grandis com podas mais leves cresceram mais que as sem poda. Logo com base nesses trabalhos pode-se verificar que diferentes espécies podem ter comportamentos diversos frente ao uso da poda radicular. E a variável altura pode ser útil para avaliar o padrão de qualidade de mudas de jatobá. Além disso, a medida da altura é de fácil determinação e não é um método destrutível (DUARTE et al., 2015).

A poda de $25,0 \%$ da raiz produziu maior DC, devido à recuperação do sistema radicular refletido no crescimento da parte aérea das plantas de jatobá. De acordo com Almeida et al. (2014), o diâmetro do coleto é um dos melhores indicadores do padrão de qualidade de mudas. Mudas com baixo valor para o DC e alturas elevadas apresentam dificuldades de se manterem eretas após o plantio (ALMEIDA et al., 2014).
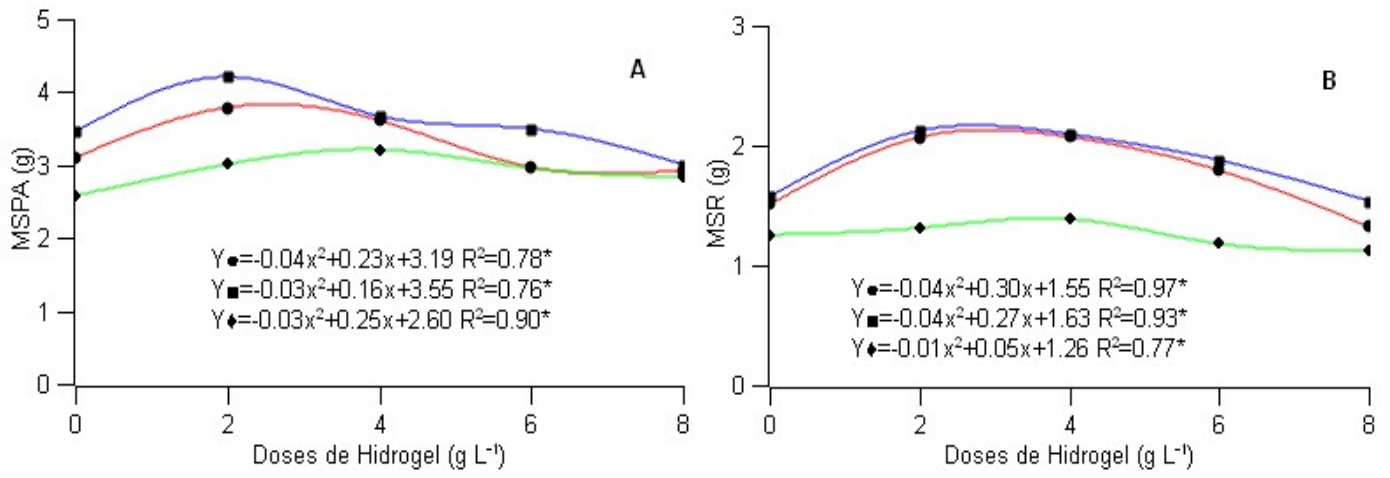

Figure 3. Comportamento das variáveis em relação aos diferentes níveis de poda radicular e doses de hidrogel em mudas de jatobá, aos 105 dias após o transplantio. Em que: massa seca da parte aérea (A) e massa seca da raiz (B). *Significativo a 5\% de probabilidade.

Figure 3. Behavior of variables in relation to the different levels of root pruning and hydrogel doses in jatoba seedlings, at 105 days after transplanting. In What: shoot dry mass (A) and root dry mass (B). * Significant at 5\% probability.
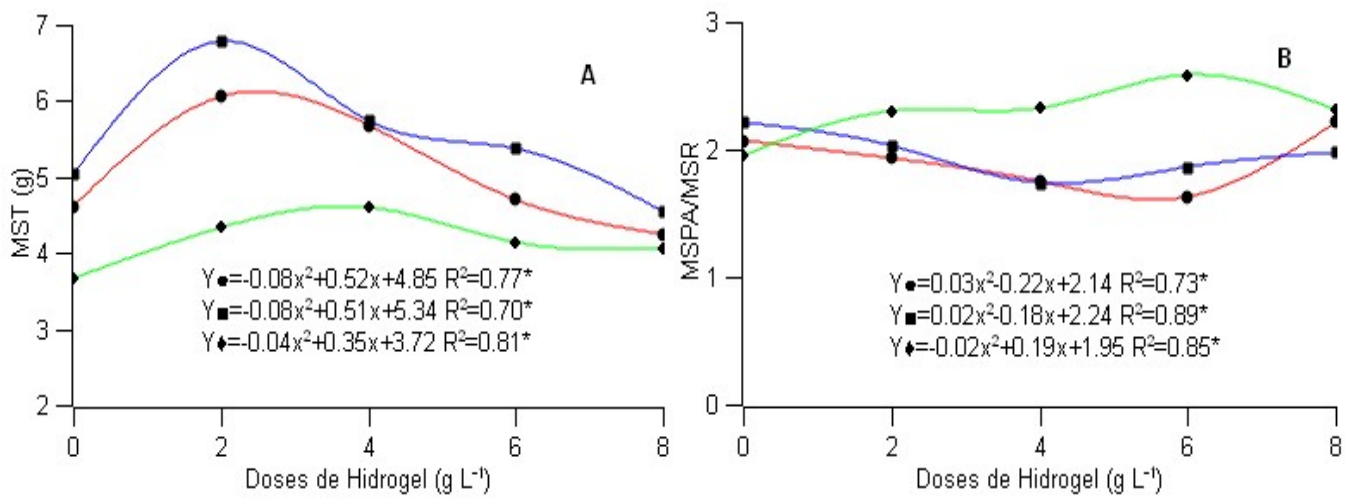

Figura 4. Comportamento das variáveis em relação aos diferentes níveis de poda radicular e doses de hidrogel em mudas de jatobá, aos 105 dias após o transplantio. Em que: massa seca total (A) e relação massa seca da parte aérea/massa seca da raiz (B). *Significativo a $5 \%$ de probabilidade.

Figure 4. Behavior of variables in relation to the different levels of root pruning and hydrogel doses in jatoba seedlings, at 105 days after transplanting. In What: total dry mass (A) and dry mass ratio of aerial part / dry mass of root (B). * Significant at 5\% probability.

No estudo, todos os tratamentos apresentaram H/DC abaixo de 10 , padrão considerado bom para mudas florestais (JOSÉ et al., 2005). A H/DC exprime o equilíbrio de crescimento, denominado de quociente de robustez, quanto maior o valor desse índice, melhor o padrão de qualidade das mudas (GOMES; PAIVA, 2011). Além disso, a H/DC reflete o acúmulo de reservas e a resistência das mudas a períodos secos, além de fixar melhor a muda no solo.

Com relação ao NF, observou-se que os resultados foram estatisticamente iguais entre si (Tabela 2). Segundo Campos et al. (2008), quanto maior o número de folhas, mais intensa é a atividade fotossintética, consequentemente, maior será o crescimento em altura e diâmetro do coleto. Portanto, neste estudo, a variável NF por si só não foi capaz de distinguir qual dos tratamentos é o mais efetivo para o desenvolvimento das mudas após o transplantio.

Os valores obtidos, para todos os tratamentos classificam as mudas como de boa qualidade em relação ao IQD. Pois todas apresentaram valor superior ao índice recomendado por Gomes; Paiva (2011). O Índice de Qualidade de Dickson (IQD) é considerado um bom indicador da qualidade de mudas, pois agrega em seu cálculo a robustez e o equilíbrio da distribuição da biomassa (AZEVEDO et al., 2010).

A capacidade de sobrevivência da muda no campo pode ser predita pela relação $\mathrm{H} / \mathrm{MSPA}$, pois quanto menor for esse índice, mais lignificada está a muda e maior a sua rusticidade. O melhor valor obtido ideal estimado para esse índice é de 2,0 sem definição de espécie. O resultado apresentado, neste cenário, difere daquele considerável desejável ou ideal, o que confere às mudas uma menor resistência aos estresses ambientais.

Mudas de jatobá tiveram um ganho em altura na dose de 2,95 $\mathrm{g} \mathrm{L}^{-1}$, o qual pode ser recomendada para diminuir gastos com água de irrigação e proporcionar mudas de boa qualidade. Os resultados deste estudo corroboram com outros trabalhos na literatura, em que o hidrogel forneceu 
maior desenvolvimento e qualidade de mudas para diferentes espécies de árvores. Por exemplo, Bernardi et al. (2012) observaram efeito positivo para altura da parte aérea com o uso de hidrogel na espécie Corymbia citridora em condições de viveiro. Navroski et al. (2015) observaram que o uso de hidrogel propiciou um ganho significativo, na altura das mudas de Eucalyptus dunnii, em comparação com a ausência do hidrogel.

O maior crescimento de DC estimado para dose 4,11 $\mathrm{g} \mathrm{L}$ $1(5,75 \mathrm{~mm})$ apresentou-se bem superior aquele apresentado pelas mudas sem hidrogel $(5,0 \mathrm{~mm})$ e isso demonstra a eficiência da presença do hidrogel em relação ao DC das mudas de jatobá. Bernardi et al. (2012) observaram que presença do hidrogel aumenta o diâmetro do coleto das mudas de Corymbia citriodora, em condições de viveiro.

Da mesma forma, o IQD, neste estudo, demonstrou que mudas de jatobá com qualidade para serem levadas a campo devem ter o hidrogel próximo a 3,89 $\mathrm{g} \mathrm{L}^{-1}$. Segundo Gomes et al. (2013) o IQD pode variar em função da espécie, do manejo das mudas no viveiro, do tipo e proporção do substrato, do volume do recipiente, com a idade em que a muda foi avaliada.

Novamente o resultado para H/MSPA foi considerado distante daquele considerável desejável. Valores para a razão $\mathrm{H} / \mathrm{MSPA}$ devem ser pequenos, pois indica maior o equilíbrio entre as partes da muda, o que confere às mudas uma maior resistência aos estresses ambientais.

Os resultados deste estudo corroboram com outros estudos na literatura, em que o hidrogel forneceu o maior desenvolvimento e qualidade para mudas de diferentes espécies de árvores. Azevedo et al. (2016) observaram que a incorporação do hidrogel, na dose de $2,0 \mathrm{~g} \mathrm{~L}^{-1}$, contribuiu para o crescimento e qualidade padrão das mudas de Corymbia citriodora Hill \& Johnson. Estes autores também afirmam que o uso de hidrogel pode variar, de acordo com a necessidade particular de cada espécie ou material genético e com as práticas de manejo adotado pelos viveristas. Segundo Navroski et al. (2015), o aumento de retenção de água, em doses superiores de hidrogel, pode causar acúmulo excessivo de água e, também, pode dificultar a absorção de nutrientes pelo sistema radicular. Esse fato pode ter sido uma das razões, para a redução nos incrementos em H, DC e IQD, encontrados neste trabalho, para doses de hidrogel maiores que $4,0 \mathrm{~g} \mathrm{~L}^{-1}$

O maior acúmulo de matéria seca da parte aérea obtida na dose de $2,75 \mathrm{~g} \mathrm{~L}^{-1}$ associado a poda mais leve foi favorecido pelo maior aproveitamento dos nutrientes e maior disponibilidade de água. Logo a combinação adequada de poda e hidrogel favorece a produção de fitomassa seca da parte aérea das mudas de jatobá. Segundo Gomes; Paiva (2011) a matéria seca da parte aérea indica a rusticidade da muda e, apesar de ser um método destrutivo, deve ser considerada durante a avaliação das características biométricas das mudas. Além disso, quanto maior o valor para a MSPA, mais lignificadas e rústicas são as mudas, logo apresentam grande potencial de sobrevivência em ambientes com condições adversas (GOMES; PAIVA, 2011).

No presente estudo, os tratamentos de hidrogel, em qualquer dose com 50,0\% de poda radicial, apresentaram menor massa seca para os sistemas radiculares. Segundo Ataíde et al. (2010), sistema radicular reduzido resulta em plantas estressadas hidricamente, por não absorverem água suficiente para balancear as perdas pela transpiração.
Também é importante que pelos absorventes da raiz, as quais assegurarão pronto crescimento radicial no campo, agilizando a adaptação da muda ao ambiente. Por outro lado, sistemas radiculares desenvolvidos possuem maior facilidade de sustentação, além de maior área e eficiência para absorção de água e nutrientes (FREITAS et al., 2009).

Quanto maior for o valor para a MST, melhor será a qualidade das mudas produzidas. Apesar de ser um método destrutivo, a biomassa seca total constitui uma boa indicação da capacidade de resistência das mudas às condições de campo (GOMES; PAIVA, 2011).

A relação MSPA/MSR é considerada um índice eficiente, pois resume, de um modo geral, a distribuição dos assimilados entre as partes da planta, ou seja, entre a parte que transpira x parte que absorve água (ALMEIDA et al., 2014). Gomes; Paiva (2011) estabelecem o valor de 2,0 à melhor relação entre a massa seca da parte aérea e a respectiva massa seca de raiz. Neste estudo, verificou-se que os valores obtidos, para MSPA/MSR das mudas de jatobá, variaram de 1,70 a 2,47, logo próximos ao estabelecido como ideal (Figura $2 \mathrm{D})$.

Desta forma, o acúmulo de matéria seca da raiz conduz o maior desenvolvimento do sistema radicular, o qual favorece a absorção de água e nutrientes e, consequentemente, o pegamento das mudas após transplantio em campo.

De maneira geral, as variáveis morfométricas e os índices obtidos neste estudo não devem ser utilizadas isoladamente para a determinação da qualidade de mudas do jatobá. Todavia o IQD, neste estudo deve ser considerado, pois no experimento demonstrou valores muito semelhantes entre os fatores isolados.

\section{CONCLUSÕES}

Poda de $25,0 \%$ do sistema radicular, favorece todos os atributos de crescimento avaliados na produção das mudas de jatobá. O uso de hidrogel nas doses entre 3,0 e 4,0 $\mathrm{g} \mathrm{L}^{-1}$, favorecem o crescimento das mudas de jatobá nos parâmetros morfométricos: H, DC, IQD e H/MSPA.

Poda de $25,0 \%$ da raiz e hidrogel nas doses entre 3,0 e 4,0 g L $\mathrm{L}^{-1}$ favorecem o crescimento das mudas de jatobá nos parâmetros morfométricos: MSPA, MSR, MST e MSPA/MSR

\section{REFERÊNCIAS}

ALMEIDA, R. S.; MAYRINCK, R. C.; ZANINI, A. M.; DIAS, B. A. S.; BARONI, G. R. Crescimento e qualidade de mudas de Croton floribundus spreng. em diferentes recipientes e substratos. Enciclopédia Biosfera, Goiânia, v. 10, n. 19, p. 672-685, 2014.

ALVARENGA, R. C.; BARROS, N. F.; DANTAS, C. E. S.; LOBÃO, D. Efeito do conteúdo de água no solo e da poda de raízes sobre o crescimento de mudas de Eucalipto. Revista Árvore, Viçosa, v. 18, n. 2, p. 107-114, 1994.

ALVAREZ, C. A.; STAPE, J. L.; SENTELHAS, P. C.; GONÇALVES, J. L. M.; SPAROVEK, G. Koppen's climate classification map for Brazil. Meteorologische Zeitschrift, Berlin, v. 22, n. 6, p. 711-728, 2013. DOI: https://dx.doi.org/10.1127/0941-2948/2013/0507

ATAÍDE, G. M.; CASTRO, R. V. O.; SANTANA, R. C.; DIAS, B. A. S.; CORREIA, A. C. G.; MENDES, A. F. N. Efeito da densidade na bandeja sobre o crescimento 
de mudas de eucalipto. Revista Trópica: Ciências Agrárias e Biológicas, Chapadinha, v. 4, n. 2, p. 21-26, 2010.

AZEVEDO, I. M. G.; ALENCAR, R. M.; BARBOSA, A. P.; ALMEIDA, N. O. Estudo do crescimento e qualidade de mudas de marupá (Simarouba amara Aubl.). Acta Amazônica, Manaus, v. 40, n. 1, p. 157-164, 2010. DOI: http://dx.doi.org/10.1590/S0044-59672010000100020

AZEVEDO, G. T. O. S.; AZEVEDO, G. B.; SOUZA, A. M.; MEWS, C. L.; SOUZA, J. R.L. Efeito de doses do hidrogel na qualidade de mudas de Corymbia citriodora Hill \& Johnson. Nativa, Sinop, v. 4, n. 4, p. 244-248, 2016. DOI: 7670.v04n04a10

BERNADI, M. R.; JÚNIOR, M. S.; DANIEL, O.; VITORINO, A. C. T. Crescimento de mudas de Corymbia citriodora em função do uso de hidrogel e adubação. Cerne, Lavras, v. 18, n. 1, p. 67-74, 2012. DOI: http://dx.doi.org/10.1590/S0104-77602012000100009

CAMPOS, M. C. C.; MARQUES, F. J.; LIMA, A. G.; MENDONÇA, R. M. N. Crescimento de porta-enxerto de gravioleira (Annona muricata L.) em substratos contendo doses crescentes de rejeitos de caulim. Revista de Biologia e Ciência da Terra, Campina Grande, v. 8, n. 1, p. 61-66, 2008.

CARRA, B.; FACHINELLO, J. C.; ABREU, E. S.; PASA, M. S.; SPAGNOL, D.; GIOVANAZ, M. A.; SILVA, C. P. Control of the vegetative growth of 'Shinseiki' pear trees by prohexadione calcium and root pruning. Pesquisa Agropecuária Brasileira, Brasília, v. 52, n. 3, p. 177-185, 2017.2 DOI: http://dx.doi.org/10.1590/s0100-204x2017000300005

CASTRO FARIA, A. B.; HIRANO, E.; PORTELA, O.; VOSGERAU, J. L.; NOGUEIRA, A. C. Poda radicial em mudas de Rheedia gardneriana. Scientia agrária, Curitiba, v. 8 , n. 1 , p. 99-101, 2007. DOI: http://dx.doi.org/10.5380/rsa.v8i1.8349

COSTA, W. S.; SOUZA, A. L.; SOUZA, P. B. Jatobá Hymenaea courbaril L.: Ecologia, Manejo, Silvicultura e Tecnologia de Espécies Nativas da Mata Atlântica. Viçosa: UFV, 2011. 21 p.

CRUZ, F. R. S.; ANDRADE, L. A.; FEITOSA, R. C. Produção de mudas de umbuzeiro (Spondias tuberosa Arruda Câmara) em diferentes substratos e tamanho de recipientes. Ciência Florestal, Santa Maria, v. 26, n. 1, p. 69-80, 2016 http://dx.doi.org/10.5902/1980509821092

DAFLON, T. M.; HÜTHER, C. M.; SANTOS, C. M. P. P.; CARVALHO, L. F.; CORREA, N. P. C.; CORREIA, D. M.; PEREIRA, C. R.; MACHADO, T. B. Incrementos na produção de biomassa total de citronela por estresse severo no sistema radicular. Revista Brasileira de Ciências Ambientais, Rio de Janeiro, n. 51, p. 95-111, 2020. DOI: http://dx.doi.org/10.5327/Z2176947820190438

DRANSKI, J. A. L.; JUNIOR, A S. P.; CAMPAGNOLO, M. A.; MALAVASI, U. C.; MALAVASI, M. M. Sobrevivência e crescimento do pinhão-manso em função do método de aplicação e formulações de hidrogel. Revista Brasileira de Engenharia Agrícola e Ambiental, Campina Grande, v. 17, n. 5, p. 537-542, 2013. DOI: http://dx.doi.org/10.1590/S141543662013000500011

DUARTE, M. L.; PAIVA, H. N.; ALVES, M. O.; FREITAS,
A. F.; MAIA, F. F.; GOULART, L. M. L. Crescimento e qualidade de mudas de vinhático (Platymenia foliolosa Benth.) em resposta à adubação com potássio e enxofre. Ciência Florestal, Santa Maria, v. 25, n. 1, p. 221-229, 2015.

FREITAS, T. A. S.; BARROSO, D. B.; SOUZA, L. S.; CARNEIRO, J. G. A. Efeito da poda de raizes sobre o crescimento das mudas de eucalipto. Ciência Florestal, Santa Maria, v. 19, n. 1, p. 1-6, 2009. DOI: https://dx.doi.org/10.5902/19805098414

GARIGLIO, M. A.; SAMPAIO, E.; CESTARO, L. A.; KAGEYAMA, P. Uso Sustentável e Conservação dos Recursos Florestais da Caatinga. Brasilia: Servico Florestal Brasileiro, 2010. 368 p.

GOMES, J. M.; PAIVA, H. N. Viveiros florestais: propagação sexuada - Série Didática. 1. ed. Viçosa, MG: UFV, 2011. $116 \mathrm{p}$.

GOMES, D. R.; CALDEIRA, M. V. W.; DELARMELINA, W. M.; GONÇALVES, E. O.; TRAZZI, P. A. Lodo de esgoto como substrato para produção de mudas de Tectona grandis L. Cerne, Lavras, v. 19, n. 1, p. 123-131, 2013. DOI: http://dx.doi.org/10.1590/S010477602013000100015

GUILHERME, M. R.; AOUADA, F. A.; FAJARDO, A. R.; MARTINS, A. F.; PAULINO, A. T.; DAVI, M. F. T.; RUBIRA, A. F.; MUNIZ, E. C. Superabsorbent hydrogels based on polysaccharides for application in agriculture as soil conditioner and nutrient carrier: a review. European Polymer Journal, New York, v. 72, p. 365-385, 2015. DOI: http://dx.doi.org/10.1016/j.eurpolymj.2015.04.017

HOSSEL, C.; OLIVEIRA, J. S. M. A.; JÚNIOR, A. W.; MAZARO, S. M.; CITADIN, I. Manejo da poda de raízes no transplantio de mudas de fruteiras nativas. Revista Brasileira de Fruticultura, Jaboticabal, v. 36, n. 3, p. 761-765, 2014. DOI: http://dx.doi.org/10.1590/0100$2945-278 / 13$

JOSÉ, A. C.; DAVIDE, A. C.; OLIVEIRA, S. L. Produção de mudas de aroeira (Schinus terebinthifolius Raddi) para recuperação de áreas degradadas pela mineração de bauxita. Cerne, Lavras, v. 11, n. 12, p. 187-196, 2005.

KLEIN, C.; KLEIN, V. A. Estratégias para potencializar a retenção e disponibilidade de água no solo. Revista Eletrônica em Gestão, Educação e Tecnologia Ambiental, Santa Maria, v. 19, n. 1, p. 21-29, 2015.

NAVROSKI, M. C.; ARAÚJO, M. M.; CUNHA, F. S.; BERGHETTI, A. L. P.; PEREIRA, M. O. Influência do polímero hidroretentor na sobrevivência de mudas de Eucalyptus dunnii sob diferentes manejos hídricos. Nativa, Sinop, v. 2, n. 2, p. 108-113, 2014. DOI: http://dx.doi.org/10.14583/2318-7670.v02n02a08

NAVROSKI, M. C.; ARAÚJO, M. M.; FLOR, C. S.; CUNHA, F. S.; BERGHETTI, A. L. P.; PEREIRA, M. O. Uso de hidrogel possibilita redução da irrigação e melhora o crescimento inicial de mudas de Eucalyptus dunnii Maiden. Scientia Forestalis, Piracicaba, v. 43, n. 106, p. 467-476, $2015 \mathrm{a}$.

NAVROSKI, M. C.; ARAÚJO, M. M.; REININGER, L. R. S.; MUNIZ, M. F. B.; PEREIRA, M. O. Influência do hidrogel no crescimento e no teor de nutrientes das mudas de Eucalyptus dunnii. Floresta, Curitiba, v. 45, n. 2, p. 315-328, 2015b. DOI: http://dx.doi.org/10.5380/rf.v45i2.34411

NEETHU, T. M.; DUBEY, P. K.; KASWALA, A. R. 
Prospects and Applications of Hydrogel Technology in Agriculture. International Journal of Current Microbiology and Applied Sciences, v. 7, n. 5, p. 31553162, 2018.2 DOI: https://dx.doi.org/10.20546/ijcmas.2018.705.369

SILVA, C. A.; NETO, D. D.; SILVA, C. J.; FREITAS, C. A. Development of Hymenaea courbaril seedlings in function of containers and irrigation blades. Revista Árvore, Viçosa, v. 40, n. 3, p. 487-498, 2016. DOI: http://dx.doi.org/10.1590/0100-67622016000300012

SOUZA, E. S.; FERNANDES, A. R.; BRAZ, A. M. S.; OLIVEIRA, F. J.; ALLEONI, L. R. F.; CAMPOS, M. C. C. Physical, chemical, and mineralogical attributes of a representative group of soils from the eastern Amazon region in Brazil. Soil, v. 4, n. 3, p. 195-212, 2018. DOI: https://dx.doi.org/10.5194/soil-4-195-2018

WATANABE, K.; SAENSUPO, S.; NA-IAM, Y.; KLOMSA-ARD, P. Effects of Superabsorbent Polymer on Soil Water Content and Sugarcane Germination and Early Growth in Sandy Soil Conditions. Sugar Technology, Amsterdam, v. 21, n. 3, p. 444-450, 2019. DOI: https://dx.doi.org/10.1007/s12355-018-0672-5

ZHAO, W.; CAO, T.; DOU, P.; SHENG, J.; LUO, M. Effect of various concentrations of superabsorbent polymers on soil particle-size distribution and evaporation with sand mulching. Scientific Reports, v. 9, n. 3511, 2019. DOI: https://dx.doi.org/10.1038/s41598-019-39412-x 